\title{
SEROPREVALENCIA DE Toxoplasma gondii EN ALPACAS DE COMUNIDADES DE LA PROVINCIA DE CANCHIS, CUSCO
}

\author{
Julia Ramírez R. ${ }^{1}$, Amanda Chávez V. ${ }^{2,3}$, Eva Casas A. ${ }^{2}$, \\ Raúl Rosadio A. ${ }^{2}$ y Néstor Falcón P. ${ }^{4}$
}

\section{Abstract}

The objective of the present study was to determine the seroprevalence of Toxoplasma gondii in alpacas of communities from the districts of Maranganí, Pitumarca, Checacupe and San Pablo, located in the province of Canchis, department of Cusco. A total of 272 blood samples were collected in March 2003, for the detection of antibodies against $T$. gondii by the indirect immunofluorescence test (IFAT). The resulting seroprevalence was $35.7 \pm 5.7 \%$, without significant differences due to district, sex, and breed; however, there was a significant association between age and IFAT value. The results of the present study agreed with other studies conducted in South American camelids in different localities in the south of Peru.

Key words: serology, toxoplasmosis, South American camelids, IFAT, Cusco

\section{RESUMEN}

El objetivo del estudio fue cuantificar la seroprevalencia de Toxoplasma gondii en alpacas de comunidades alpaqueras de los distritos de Maranganí, Pitumarca, Checacupe y San Pablo, en la provincia de Canchis, Cusco. Se recolectaron 272 muestras de sangre en marzo del 2003 para la detección de anticuerpos contra T. gondiimediante la prueba de inmunofluorescencia indirecta (IFI). Se encontró una seroprevalencia moderada de $35.7 \pm$ $5.7 \%$. No se encontró asociación entre las variables distrito, sexo, raza y la respuesta a la prueba de IFI. Sin embargo, se encontró una asociación significativa entre la edad y la respuesta a la prueba. La seroprevalencia del presente estudio concuerda con resultados obtenidos en camélidos sudamericanos en otras zonas del sur del Perú.

Palabras clave: serología, toxoplasmosis, camélidos sudamericanos, IFI, Cusco

\footnotetext{
${ }^{1}$ Práctica privada

${ }^{2}$ Laboratorio de Microbiología y Parasitología Veterinaria, FMV-UNMSM

${ }^{3}$ E-mail:a_chavez_g@hotmail.com

${ }^{4}$ Laboratorio de Medicina Veterinaria Preventiva, FMV-UNMSM
} 


\section{INTRODUCCIÓN}

La alpaca es una especie nativa de importancia social y económica, debido a que miles de familias de la región andina del sur del Perú se dedican a su crianza y explotación. La producción de estos animales se encuentra limitada por diversos problemas sanitarios y reproductivos, dentro de los cuales las enfermedades parasitarias, como la toxoplasmosis, constituyen uno de los principales problemas en pequeños rumiantes (ovinos y caprinos), ya que causan abortos y muerte (Acha y Szyfres, 2003; Ramírez, 1991). Así mismo, se le considera una de las infecciones parasitarias zoonóticas más difundidas en el mundo (Acha y Szyfres, 2003). Por otro lado, es importante recalcar que las comunidades campesinas se caracterizan por tener un tipo de crianza mixta, donde la convivencia de ovinos, bovinos y alpacas favorecería la difusión y transmisión de enfermedades.

La información disponible sobre toxoplasmosis en camélidos sudamericanos en el país consiste en estudios serológicos realizados en diferentes empresas alpaqueras, que demuestran seroprevalencias moderadas y altas a $T$. gondii; sin embargo son escasos los reportes de esta enfermedad en comunidades campesinas, las cuales manejan cerca del $90 \%$ de la población de alpacas y casi la totalidad de llamas (Fernández-Baca, 1991). En base a esto, se planificó el presente estudio con el objetivo de cuantificar la seroprevalencia de $T$. gondii en alpacas de varias comunidades de la provincia de Canchis, Cusco.

\section{Materiales y Métodos}

\section{Lugar de estudio}

La recolección de muestras se llevó a cabo en marzo del 2003 en las comunidades de los distritos de Maranganí (Cheraje, Chillihua, Fundo Tatum Rumi, Jancoca, Silli),
Checacupe (Phatanca, Llutuyo, Yanacucho, Cerro Azuchecacupe), Pitumarca (Japura, Pinaya, Phusa) y San Pablo (Callanca), pertenecientes a la provincia de Canchis, departamento de Cusco, y que se localizan a 4,200 msnm.

\section{Animales y tamaño muestral}

Las alpacas pertenecían a pequeños productores, fueron criadas en forma extensiva sobre pastos naturales, y sin identificación individual en su mayoría. Los animales se clasificaron en base a las variables sexo, raza (Suri y Huacaya) y edad (calculada a través de la erupción dentaria).

El cálculo del tamaño muestral se hizo utilizando la información distrital del III Censo Nacional Agropecuario (INEI, 1994), que indica una población de 48,882 alpacas en Maranganí, 34,090 en Pitumarca, 19,862 en Checacupe y 16,049 en San Pablo (población total: 118,883 alpacas). Por tratarse de una población muy grande, en el cálculo del tamaño muestral se utilizó la fórmula de comprobación de una proporción para poblaciones infinitas (Daniel, 1996), resultando una muestra de 272 animales. Adicionalmente se realizó una estratificación por distritos.

\section{Recolección de muestras y detección de anticuerpos}

Se recolectaron muestras de sangre mediante punción de la vena yugular. Las muestras se centrifugaron y bs sueros resultantes se conservaron en congelación a $-20^{\circ} \mathrm{C}$ hasta su posterior procesam iento en el Laboratorio de Parasitología de la Facultad de M edicina Veterinaria de la Un iversidad $\mathrm{Na}-$ cional M ayor de San M arcos (FM V U N M S M ).

Se utilizó la prueba de inmunofluorescencia indirecta (IFI) para la detección de anticuerpos contra $T$. gondii (Trees et al., 1993), pero modificada en los laboratorios de parasitología de la Universidad 
Cuadro 1. Seroprevalencia de Toxoplasma gondii en alpacas de comunidades campesinas de la provincia de Canchis, Cusco (2003)

\begin{tabular}{cccc}
\hline \multirow{2}{*}{ Distrito } & \multicolumn{2}{c}{ Animales } & Prevalencia $\pm \mathrm{IC}^{1}$ \\
\cline { 2 - 3 } & Muestreados (n) & Positivos (n) & $(\%)$ \\
\hline Maranganí & 112 & 38 & $33.9 \pm 8.8$ \\
Pitumarca & 78 & 34 & $43.6 \pm 11.0$ \\
Checacupe & 45 & 14 & $31.1 \pm 13.5$ \\
San Pablo & 37 & 11 & $29.7 \pm 14.7$ \\
\hline \multicolumn{1}{c}{ Total } & 272 & 97 & $35.7 \pm 5.7$ \\
\hline${ }^{1}$ Intervalo de confianza del 95\% & & &
\end{tabular}

Complutense de Madrid y de la FMVUNMSM. Se utilizó una dilución del suero de $1 / 50$.

\section{Análisis estadístico}

Los resultados obtenidos fueron expresados en forma porcentual, utilizando la fórmula de prevalencia, con sus respectivos intervalos de confianza (IC) y con un nivel de confianza del 95\%. Además, se estableció la relación entre los niveles de seroprevalencia con las variables distrito, edad, sexo y raza mediante la prueba de regresión logística.

\section{Resultados y Discusión}

La seroprevalencia de T. gondii en alpacas de las comunidades de cuatro distritos de la provincia de Canchis, Cusco, determinada mediante la técnica de inmunfluorescencia indirecta (IFI) fue de $35.7 \pm$ $5.7 \%$ (Cuadro 1), sin que hubiera diferencias estadísticas entre distritos ( $p>0.05$ ). El resultado fue de un valor medio al indicado por otros autores. Por ejemplo, Góngora (1992) encontró una seroprevalencia de $24 \%$ en Puno usando la misma técnica, en tanto que Gómez et al. (2003) y Leguía et al. (1987) reportan valores de 44 a $50 \%$ con la ténica de hema- glutinación indirecta (HAI). Por otro lado, estudios realizados en la SAIS Túpac Amaru, Junín (Poma, 2003), señalan seroprevalencias de $23 \%$. Entre los factores que determinarían la fluctuación de estos valores se puede mencionar las características medioambientales de la zona en estudio, la altitud sobre el nivel del mar, la presencia del hospedero definitivo y el manejo, así como la técnica diagnóstica utilizada (Marcas et al., 2004; Gómez et al., 2003).

Los datos encontrados hasta el momento muestran que la seroprevalencia de este parásito en la zona sur del país es mayor que en la sierra central, probablemente debido a la existencia de un ambiente más contaminado, ya sea por la existencia de un mayor número de hospederos definitivos o debido a que la zona de estudio corresponde a una puna semihúmeda, condición importante para la supervivencia de los ooquistes infectivos en el medio ambiente, ya que éstos pueden permanecer viables por varios meses en ambientes fríos y húmedos (Acha y Szyfres, 2003). También se debe mencionar que la mayoría de estudios sobre la seroprevalencia de $T$. gondii en camélidos sudamericanos ha sido realizada con la técnica de HAI, que presenta una sensibilidad y especificidad menor que la IFI. 
Cuadro 2. Seroprevalencia ${ }^{1}$ de Toxoplasma gondii en alpacas, según edad, sexo y raza, en la provincia de Canchis, Cusco (2003)

\begin{tabular}{cccccccc}
\hline \multirow{2}{*}{$\begin{array}{c}\text { Edad } \\
\text { (años) }\end{array}$} & \multicolumn{2}{c}{ S e x o } & & \multicolumn{2}{c}{ Raza } & & \\
\cline { 8 - 9 } \cline { 5 - 7 } & Hembra & Macho & & Huacaya & Suri & & $\begin{array}{c}\text { Positivos } \\
\pm \text { IC }^{2}\end{array}$ \\
\hline 2 & $7 / 35$ & $1 / 5$ & & $8 / 33$ & $0 / 7$ & $8 / 40$ & $20.0 \pm 12.4$ \\
4 & $22 / 63$ & $0 / 2$ & & $18 / 52$ & $4 / 13$ & $22 / 65$ & $33.8 \pm 11.5$ \\
$\geq 6$ & $61 / 144$ & $6 / 23$ & & $53 / 129$ & $14 / 38$ & $67 / 167$ & $40.1 \pm 7.4$ \\
\hline \multirow{2}{*}{ Total } & $90 / 242$ & $7 / 30$ & & $79 / 214$ & $18 / 58$ & $97 / 272$ & $35.7 \pm 5.7$ \\
& $37.2 \pm 6.1$ & $23.3 \pm 15.1$ & & $36.9 \pm 6.5$ & $31.1 \pm 11.9$ & & \\
\hline
\end{tabular}

${ }^{1}$ Positivo/total

2 Intervalo de confianza del 95\%

Cuadro 3. Evaluación de las variables distrito, edad, sexo y raza como factores de riesgo para la infección por Toxoplasma gondii en alpacas de la provincia de Canchis, Cusco (2003)

\begin{tabular}{clcccc}
\hline \multirow{2}{*}{ Variable } & $\begin{array}{c}\text { Nivel de } \\
\text { significancia }\end{array}$ & $\begin{array}{c}\text { Odds Ratio } \\
(\text { OR })\end{array}$ & \multicolumn{2}{c}{$\begin{array}{c}\text { IC del 95 \% del OR } \\
\text { Mínimo }\end{array}$} & Máximo \\
\hline \multirow{3}{*}{ Distrito } & San Pablo & & 1 & ----- & ----- \\
& Checacupe & 0.560 & 1.347 & 0.495 & 3.666 \\
& Maranganí & 0.510 & 1.320 & 0.578 & 3.015 \\
& Pitumarca & 0.112 & 2.022 & 0.879 & 4.814 \\
& 2 años & & 1 & ----- & ----- \\
\multirow{2}{*}{ Edad } & 4 años & 0.242 & 1.760 & 0.683 & 4.534 \\
& $\geq 6$ años & 0.022 & 2.691 & 1.156 & 6.266 \\
Sexo & Machos & & 1 & ----- & ----- \\
& Hembras & 0.098 & 2.200 & 0.865 & 5.591 \\
\multirow{2}{*}{ Raza } & Suri & & 1 & ----- & ----- \\
& Huacaya & 0.726 & 1.129 & 0.573 & 2.223 \\
\hline
\end{tabular}

Se ha observado una relación directamente proporcional entre edad y porcentaje de anticuerpos contra $T$. gondii. Alpacas de 2,4 y 6 años presentaron seroprevalencias de 20, 34 y 40\%, respectivamente (Cuadro 2). Esto se explicaría porque los animales de mayor edad han tenido más posibilidades de contacto con el parásito al haber estado expuestos por mayor tiempo al consumo de agua y pastos contaminados con ooquistes infectivos. Esto concuerda con otros resultados en alpacas de 0 a 1 y 3 años con 30 y $62 \%$ de seroprevalencia, respectivamente (Gómez et al., 2003); sin embargo, en este estudio no se 
encontraron diferencias estadísticas debido a sexo o raza ( $p>0.05)$. No obstante, es importante destacar que en hembras existe la condición del relajamiento inmuneperiparto, que las harían más susceptibles que a los machos frente a una infección.

Los resultados del presente estudio fueron similares a otros reportes de la zona de Puno en llamas (Marcas et al., 2004; Gómez et al., 2003). Estudios referentes a la seroprevalencia de $T$. gondii no sólo se han limitado a camélidos domésticos como la alpaca y la llama, sino también a especies silvestres como la vicuña. Pastor et al. (2003) encontraron en Puno, mediante la técnica de HAI, una prevalencia de $14.9 \%$ en estos animales, un valor bajo si se le compara con resultados de estudios en alpacas y llamas, posiblemente debido a que su hábitat se encuentre entre los 4,200 y 5,000 msnm donde las condiciones climáticas no favorecen la supervivencia de los ooquistes.

En la evaluación de las variables distrito, edad, sexo y raza (Cuadro 3), se observa que sólo la edad representó factor de riesgo, toda vez que la posibilidad de infección en el grupo de 4 años con respecto al de 2 años fue de $1.76 \mathrm{y}$ en el grupo $\geq 6$ años fue de 2.69 .

\section{Conclusiones}

- La seroprevalencia de Toxoplasma gondii en alpacas de comunidades de los distritos de la provincia de Canchis, departamento de Cusco, fue moderada $(35.7 \pm 5.7 \%)$.

- La variable edad representó un factor de riesgo para la infección por $T$. gondii en alpacas, en tanto que las variables distrito, sexo y raza no fueron de importancia.

\section{Literatura Citada}

1. Acha, P.; B. Szyfres. 2003. Zoonosis y enfermedades transmisibles comunes al hombre y a los animales. $3^{\mathrm{a}}$ ed. p 88-97. Vol. III. Parasitosis. Publicación Científica y Técnica $\mathrm{N}^{\circ} 580$. OPS. Lima.

2. Daniel, W. 1996. Bioestadística: base para el análisis de las ciencias de la salud. $5^{a}$ ed. p 205-207. Ed. Limusa. México.

3. Dubey, J.; L. Rickard; G. Zimmerman; D. Mulrooney. 1992. Seroprevalence of Toxoplasma gondii in llamas (Lama glama) in the northwest USA. Vet. Parasitol. 44: 295-298.

4. Fernández-Baca, S. 1991. Avances y perspectivas del conocimiento de los camélidos sudamericanos. FernándezBaca, S. (ed). p 9-10. FAO. Santiago de Chile.

5. Gómez, F.; A. Chávez; E. Casas; E. Serrano; O. Cárdenas. 2003. Determinación de la seroprevalencia de toxoplasmosis en alpacas y llamas en la Estación Experimental INIA-PUNO. Rev. Inv. Vet. Perú 14: 49-53.

6. Góngora, M. 1992. Prevalencia de anticuerpos contra Toxoplasma gondii en las comunidades alpaqueras de Vilcallamas, Bajo Llallagua, Huanacayama y Llusta. Tesis de Médico Veterinario Zootecnista. Facultad de Medicina Veterinaria y Zootecnia, Univ. Nacional del Altiplano. Puno. $47 \mathrm{p}$.

7. INEI. 1994. III Censo Nacional Agropecuario (CENAGRO). Banco de datos estadísticos. Disponible en: htpp: www.inei.gob.pe

8. Leguía, G.; H. Samamé; C. Guerrero; M. Rojas; A. Núñez. 1987. Prevalencia de anticuerpos contra Toxoplasma gondii en alpacas. Rev. Cien. Vet. Lima 3: 19-21.

9. Marcas, G.; A. Chávez; E. Casas; W. García; N. Falcón. 2004. Seroprevalencia de Toxoplasma gondii en llamas de dos fundos ganaderos de la provincia de Melgar, Puno. Rev. Inv. Vet. Perú 15: 44-48. 
10. Pastor, J.; A. Chávez; E. Casas; E. Serrano. 2003. Seroprevalencia de Toxoplasma gondii en vicuñas de Puno. Rev. Inv. Vet. Perú 14: 79-82.

10. Poma, E. 2003. Seroprevalencia de anticuerpos contra Toxoplasma gondii en alpacas (Lama pacos) de la unidad de producción de Cochas de la SAIS Túpac Amaru. Tesis de Médico Veterinario. Facultad de Medicina Veterinaria, Univ. Nacional Mayor de San Marcos. Lima. 56 p.
11. Ramírez, A. 1991. Enfermedades infecciosas. En: Avances y perspectivas del conocimiento de los camélidos sudamericanos. Fernández-Baca, S. (ed). p 334337. FAO. Santiago, Chile.

12. Trees, A.; F. Guy; B. Tennant; A. Balfour; J.P. Dubey. 1993. Prevalence of antibodies to Neospora caninum in a population of urban dogs in England. Vet. Rec. 132: 125-126. 